\title{
Economic Dispatch with Multiple Fuel Options Using CCF
}

\author{
Radhakrishnan Anandhakumar, Srikrishna Subramanian \\ Department of Electrical Engineering, FEAT, Annamalai University, \\ Annamalainagar, India \\ E-mail: anandr1979@yahoo.com,drsmani@yahoo.co.in \\ Received February 6, 2011; revised March 24, 2011; accepted April 2, 2011
}

\begin{abstract}
This paper presents an efficient analytical approach using Composite Cost Function (CCF) for solving the Economic Dispatch problem with Multiple Fuel Options (EDMFO). The solution methodology comprises two stages. Firstly, the CCF of the plant is developed and the most economical fuel of each set can be easily identified for any load demand. In the next stage, for the selected fuels, CCF is evaluated and the optimal scheduling is obtained. The Proposed Method (PM) has been tested on the standard ten-generation set system; each set consists of two or three fuel options. The total fuel cost obtained by the PM is compared with earlier reports in order to validate its effectiveness. The comparison clears that this approach is a promising alternative for solving EDMFO problems in practical power system.
\end{abstract}

Keywords: Economic Load Dispatch, Composite Cost Function, Multiple Fuel Options, Piecewise Quadratic Function, Mathematical Model

\section{Introduction}

The economic dispatch problem in a power system is to determine the optimal combination of power outputs for all generating units which will minimize the total cost while satisfying the load and operational constraints [1]. The economic dispatch problem is very complex to solve because of its colossal dimension, a non-linear objective function, and a large number of constraints. Conventional techniques lambda iteration method and quadratic programming offer good results, but when the search space is nonlinear and has discontinuities, they become very complicated with a slow convergence ratio and do not always seek the optimal solution. New numerical methods are needed to cope with these difficulties, especially those with high speed search for the optimal and not being trapped in local minima [2].

The stochastic search algorithms such as Simulated Annealing (SA) and Genetic Algorithm (GA) have been applied to determine the optimal generation schedule for economic dispatch problem in a power system [3]. SA is applied in many power system problems, but setting the control parameters of the SA algorithm is a difficult task, and the convergence speed is slow when applied to a real system. The GA methods have been employed to successfully to solve complex optimization problem, recent research has identified some deficiencies in GA per- formance. Particle swarm optimization method (PSO) has been applied for solving economic dispatch problems with various operating constraints [4].

A novel optimization approach, Artificial Immune System (AIS) has been applied to solve constrained economic load dispatch problem [5]. This approach utilizes the clonal selection principle and evolutionary approach wherein cloning of antibodies is performed followed by hyper mutation. A novel coding scheme for practical economic dispatch by modified particle swarm optimization approach has also been proposed to solve economic dispatch problem [6]. The heuristic search technique, Differential Evolution has been suggested for solving economic dispatch problems [7]. Bacterial ForagingNelder Mead method has been applied for the solution of economic dispatch problems [8].

In certain fossil fire systems, the generation cost function is represented as a segmented piecewise quadratic function. The generating unit, supplied with multi-fuel sources like coal, natural gas or oil suffers with the problem of determining the most economic fuel to burn. Such a problem has been solved using the Hierarchical Method (HM) of Lagrangian multipliers method to find the incremental fuel cost for subsystems comprising sets of units [9]. The solution searches for the optimal for various choices of fuel and generation range of the units iteratively. A Hopfield neural network approaches to 
economic dispatch problems has been proposed [10]. An improved adaptive Hopfield Neural Network (HNN) approach has been proposed for finding the solution for economic dispatch with multiple fuel options [11]. The HNN suffers with slow convergence rate and normally takes a large number of iterations. A hybrid real coded GA method has been presented for solving the economic dispatch problem with multiple fuel options [12].

An enhanced Lagrangian neural network has been applied to solve the economic load dispatch problems with piecewise quadratic cost functions [13]. In this method the convergence speeds are enhanced by employing by momentum technique and providing criteria for choosing the learning rate. Economic dispatch solutions with piecewise quadratic cost functions has been solved by using improved genetic algorithm [14]. In order to improve the effectiveness of genetic algorithm multi-stage algorithm and directional crossover methods are proposed and projection method is introduced to satisfy a linear equality constraint from power balance. The heuristic search techniques such as PSO [15] ,Taguchi method (TM) [16], Evolutionary Programming (EP) [17] and its improved version are also been applied to solve the economic dispatch problems with multiple fuel options $[18,19]$.

The CCF is a non-iterative direct method, gives the most economic dispatches of the online units with less computation time. In this paper, the CCF is used to solve the economic dispatch problem with multiple fuel options.

\section{Problem Formulation}

\subsection{Nomenclature}

$\begin{array}{ll}a_{i}, b_{i}, c_{i} & \begin{array}{l}\text { Fuel cost coefficients of the units } \\ a_{s j}, b_{s j}, c_{s j}\end{array} \\ A, B, C & \begin{array}{l}\text { Fuel cost coefficients of the set } S \text { with } k \\ \text { fuel options }\end{array} \\ A_{S}, B_{S}, C_{S} & \text { Composite cost coefficients } \\ A_{P}, B_{P}, C_{P} & \text { Composite cost coefficients of the set } S \\ F C_{S j} & \text { Fuel cost function of the set } S \text { with } k \text { fuel } \\ F C_{S} & \text { options, in } \$ / \mathrm{h} \\ F C_{P} & \text { Fuel cost function of the set } S \text {, in } \$ / \mathrm{h} \\ k & \text { Fuel cost function of the plant, in } \$ / \mathrm{h} \\ N & \text { Number of fuel options in a plant } \\ P_{S}^{\min } & \text { Number of generation units } \\ P_{S}^{\max } & \text { Minimum power generation of set } S \text {, in } \\ P_{S} & \text { MW } \\ P_{G} & \text { Maximum power generation of set } S \text {, in } \\ & \text { MW }\end{array}$

$\begin{array}{ll}P_{D} & \text { Power demand, in MW } \\ S & \text { Set of generating units in a plant } \\ \lambda & \text { Incremental production cost, in } \$ / \mathrm{MWh}\end{array}$

\subsection{Economic Dispatch Problem with Multiple Fuels}

The main objective of economic dispatch is to find the optimal combination of power generation that minimizes the total generation cost while satisfying equality and inequality constraints. A piecewise quadratic function is used to represent the input-output curve of a generator with multiple fuel options. For a generator with $k$ fuel options, the cost curve is divided into $k$ discrete regions between lower and upper bounds. The economic dispatch problem with piecewise quadratic function is defined as

$$
\begin{aligned}
& \text { Minimize } \sum_{i=1}^{N} F_{i}\left(P_{i}\right) \\
& F_{i}\left(P_{i}\right)=\left\{\begin{array}{ccc}
a_{i 1} P_{i}^{2}+b_{i 1} P_{i}+c_{i 1}, & \text { fuel } 1, & P_{i}^{\min } \leq P_{i} \leq P_{i 1} \\
a_{i 2} P_{i}^{2}+b_{i 2} P_{i}+c_{i 2}, & \text { fuel } 2, & P_{i 1} \leq P_{i} \leq P_{i 2} \\
\vdots & & \\
a_{i k} P_{i}^{2}+b_{i k} P_{i}+c_{i k}, & \text { fuel } k, & P_{i k-1} \leq P_{i} \leq P_{i}^{\max }
\end{array}\right.
\end{aligned}
$$

where $F_{i}\left(P_{i}\right)$ is the fuel cost function of the $i^{\text {th }}$ unit, $P_{i}$ is the power output of the $i^{t h}$ unit, $\mathrm{N}$ is the number of generating units in the system and $a_{i k}, b_{i k}$ and $c_{i k}$ are cost coefficients of the $i^{\text {th }}$ unit using fuel type $k$.

Minimization of the generation cost is subjected to the following constraints:

1) The power balance constraints

$$
\sum_{i=1}^{N} P_{i}=P_{D}
$$

where $P_{D}$ is the total system demand in MW.

2) Generating capacity constraints

$$
P_{i}^{\min } \leq P_{i} \leq P_{i}^{\max }
$$

where, $P_{i}^{\min }$ and $P_{i}^{\max }$ are the minimum and maximum power outputs of the $i^{\text {th }}$ unit.

\section{Composite Cost Function (CCF)}

The composite cost coefficients were reported in the literature [9].

$$
\begin{gathered}
A=1 /\left(1 / a_{1}+1 / a_{2}+\cdots+1 / a_{N}\right) \\
B=\left(b_{1} / a_{1}+b_{2} / a_{2}+\cdots+b_{N} / a_{N}\right) A \\
2 A P_{G}+B=\lambda ; \text { where } P_{G}=P_{D} \\
P_{G i}=\frac{\lambda-b_{i}}{2 a_{i}} \text { where } i=1,2, \cdots, N
\end{gathered}
$$




$$
\begin{gathered}
C=\left(c_{1}+c_{2}+\cdots+c_{N}\right) \\
-\left(b_{1}^{2} / 4 a_{1}+b_{2}^{2} / 4 a_{2}+\cdots+b_{N}^{2} / 4 a_{N}\right)+B^{2} / 4 A \\
F C_{P}=A_{P} P_{G}^{2}+B_{P} P_{G}+C_{P}
\end{gathered}
$$

The $A, B$ and $C$ are the composite cost coefficients and can be easily calculated by using Equations (4), (5) and (7) respectively. For a particular load demand, the optimal generation of units is directly computed using Equation (6).

\section{Proposed Approach for Economic Dispatch with MFO}

The proposed methodology consist two stages of the most economic fuel identification and economic scheduling. In the first stage, the composite cost function of the plant is developed as detailed in the previous section. The incremental cost of the plant for a particular load demand is calculated and the generation dispatch for each unit is determined. The dispatch of each set directly indicates the most economical fuel and feasible operating region. In the second stage, the dispatch of the generating units is refined within the feasible operating region. The composite cost function is developed with the selected fuels and is solved to obtain the most economic dispatch of generating units.

\subsection{Identification of the Most Economic Fuel}

Consider a plant consists of ' $S$ ' set of generation, each set consists of ' $k$ ' fuel options.

The fuel cost function of the set ' $S$ ' with ' $k$ ' fuel options is,

$$
\begin{aligned}
& F C_{S j}=a_{S j} P_{S}^{2}+b_{S j} P_{S}+c_{S j}, \\
& P_{S}^{\min } \leq P_{S} \leq P_{S}^{\max } ; \\
& S=1,2, \cdots, N ; j=1,2, \cdots, k
\end{aligned}
$$

The composite cost function of set ' $S$ ' is calculated by using Equations (4), (5) and (7).

$$
\begin{gathered}
F C_{S}=A_{S} P_{S}^{2}+B_{S} P_{S}+C_{S} \\
A_{S}=1 /\left(1 / a_{1}+1 / a_{2}+\cdots+1 / a_{k}\right) \\
B_{S}=\left(b_{1} / a_{1}+b_{2} / a_{2}+\cdots+b_{k} / a_{k}\right) A_{S} \\
C_{S}=\left(c_{1}+c_{2}+\cdots+c_{k}\right) \\
-\left(b_{1}^{2} / 4 a_{1}+b_{2}^{2} / 4 a_{2}+\cdots+b_{k}^{2} / 4 a_{k}\right)+B_{S}^{2} / 4 A_{S}
\end{gathered}
$$

In this manner, the CCF for the plant is calculated and is given as,

$$
F C_{P}=A_{P} P_{G}^{2}+B_{P} P_{G}+C_{P}
$$

The composite coefficients are constant for any load demands.

The incremental production cost of the plant for the demand is calculated by

$$
\lambda=2 A_{P} P_{G}+B_{P} \text {; where } P_{G}=P_{D}
$$

The economic dispatch of the set ' $S$ ' is calculated as,

$$
P_{S}=\frac{\lambda-B_{S}}{2 A_{S}}
$$

The above equation provides the dispatch of each set to meet the load demand. Based on this dispatch, the most economical fuel and the feasible operating region of each set can be easily identified.

\subsection{Economic Dispatch of the Selected Fuels}

The composite cost function is developed using the most economic fuels. The generation dispatch is refined within the feasible limits as detailed in Section 3.

The computational flow of the proposed methodology for solving economic dispatch problem with multiple fuel options is presented as a flow chart in Figure 1.

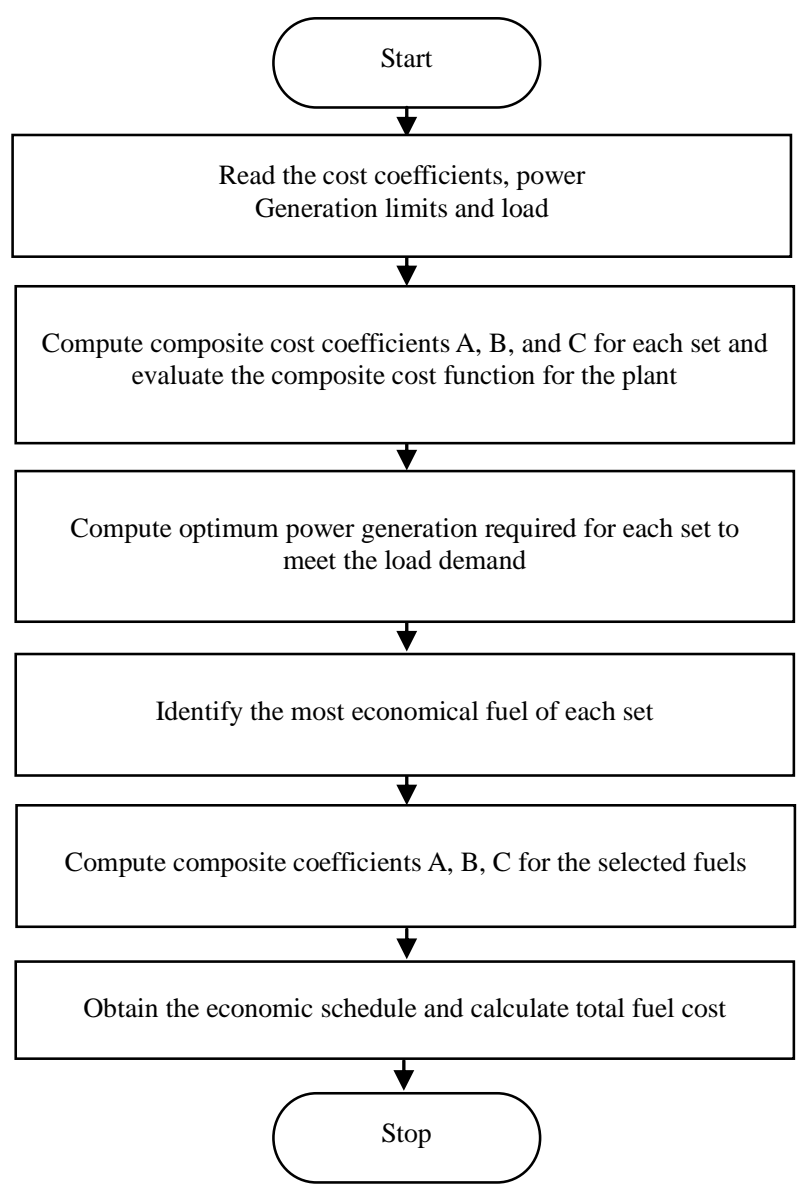

Figure 1. Flow chart of the proposed method. 


\section{Numerical Simulation Results and Discussion}

The proposed technique has been implemented in MATLAB on a $2.10 \mathrm{GHz}$ core2Duo processor PC. The simulation studies have been carried out on ten-generating unit system with multiple fuel options [9]. This problem includes one objective function with ten variable parameters $\left(P_{1}, P_{2}, \cdots, P_{10}\right)$, one equality and twenty inequality constraints, i.e. power balance constraint and maximum and minimum limits of each generating unit.

By the proposed strategy, the economic dispatch solution for the given system can be obtained in two stages: 1) evaluate the composite fuel cost function for the plant and is solved to identify the most economic fuel and feasible operating region of each set, and 2) the optimal dispatches are calculated by solving composite fuel cost function with selected fuels.

The implementation of the proposed strategy for the given system is detailed as follows. The equivalent cost function of set ' $S$ ' is calculated using the Equations (11), (12) and (13). In the selected sample system the number of sets in the plant is ten and the number of units is twenty nine.

For example, the equivalent cost function of the set 5 is,

$$
F C_{5}=0.000044807 P_{5}^{2}-0.0493 P_{5}+91.5841
$$

Similarly, for set 8, the equivalent cost function is,

$$
F C_{8}=0.000067369 P_{8}^{2}-0.04404 P_{8}+126.6098
$$

In this manner, the equivalent cost function of each set is determined. By combining these cost coefficients, the equivalent cost function of the plant is calculated.

The equivalent cost function of the plant is,

$$
F C_{P}=7.3287 e^{-06} P_{G}^{2}+0.3933 P_{G}-1518
$$

The incremental cost of the plant is obtained by using Equation (15).

For a load demand of $2400 \mathrm{MW}$, the $\lambda$ is 0.4285 and the dispatch of each set is determined by using Equation (16). Then, the dispatches of the set 5 and 8 are $278 \mathrm{MW}$ and $265 \mathrm{MW}$ respectively. In this manner, the dispatch of each set is identified and it indicates the most economical fuel and the feasible operating region. For the set 5, the dispatch is $278 \mathrm{MW}$, then the most economical fuel is 1 and the feasible operating region is $190 \mathrm{MW}$ to $338 \mathrm{MW}$. For the set 8 , the dispatch is $265 \mathrm{MW}$, then the most economical fuel is 3 and the feasible operating region is $200 \mathrm{MW}$ to $265 \mathrm{MW}$.

Similarly, the economic dispatch is performed to determine the optimal dispatches within the feasible oper- ating region by using the composite cost function of the selected fuels. During the calculation of dispatch, if the generation of any unit violates the effective limits, its generation are fixed at the violated limit. Then that unit is eliminated from the dispatch procedure. The generation of all the units except the violated unit is recalculated using the above procedure with total generation equal to the load demand minus the generation of the limit violated unit.

The simulation is performed for various load demands of $2400 \mathrm{MW}, 2500 \mathrm{MW}, 2600 \mathrm{MW}$ and $2700 \mathrm{MW}$. The optimal dispatches obtained by the proposed methodology and HM [9] for the above mentioned load demands are compared and the comparison is presented in Table $\mathbf{1 .}$ These results signify that the proposed CCF always provides better solution than HM [9]. Though the HM method is an iterative mathematical approach, suffers with the assumption of initial lambda values. In addition, two operating points having same incremental cost also exist and it requires valid assumption to choose the optimal fuel for a particular demand, improper selection will lead infeasible solution. Additionally, the proposed CCF is a direct or non iterative method, it does not demand any initial guess values for economic dispatch of units for the given load demand.

The comparison of total fuel cost obtained by proposed methodology, HM [9], HNN [10], AHNN [11], HGA [12], Modified PSO (MPSO) [15], TM [16], Improved Fast EP (IFEP) [17], Fast EP (FEP) [17], Classical EP (CEP) [17], PSO [18], and Improved EP (IEP) [19] is presented in Table 2 . As seen the comparison, the generation costs obtained by CCF are lowest among the results. Moreover, the optimal fuel cost obtained through the CCF method is exactly same as the HGA [12], except the load demand of 2600 MW. However, the proposed method directly provides the optimal schedule and it utilizes the CCF for fuel selection and economic scheduling. These comparison results confirm that the CCF provides better solution quality.

The salient features of proposed approach over existing methods are:

- The approach is conceptually simple.

- It is a non iterative method.

- The simplified generalized expression directly gives the most economical fuel and the feasible operating region for a particular load demand.

- It also provides the most economic schedule of generation with less computational effort.

- It requires negligible computational time, hence it is suitable for on line applications.

- The performance of the proposed is independent of system size; hence it is suitable for system of any size. 
Table 1. Comparison of simulation results between proposed method and HM.

\begin{tabular}{|c|c|c|c|c|c|c|c|c|}
\hline \multirow{3}{*}{ UNIT } & \multicolumn{4}{|c|}{ LOAD DEMAND = $2400 \mathrm{MW}$} & \multicolumn{4}{|c|}{ LOAD DEMAND = $2500 \mathrm{MW}$} \\
\hline & \multicolumn{2}{|r|}{ PM } & \multicolumn{2}{|c|}{ HM [9] } & \multicolumn{2}{|r|}{ PM } & \multicolumn{2}{|c|}{ HM [9] } \\
\hline & FT & GEN (MW) & FT & GEN (MW) & FT & GEN (MW) & FT & GEN (MW) \\
\hline 1 & 1 & 189.7405 & 1 & 193.2 & 2 & 206.5190 & 2 & 206.6 \\
\hline 2 & 1 & 202.3427 & 1 & 204.1 & 1 & 206.4573 & 1 & 206.5 \\
\hline 3 & 1 & 253.8953 & 1 & 259.1 & 1 & 265.7391 & 1 & 265.9 \\
\hline 4 & 3 & 233.0456 & 3 & 234.3 & 3 & 235.9531 & 3 & 236.0 \\
\hline 5 & 1 & 241.8297 & 1 & 249.0 & 1 & 258.0177 & 1 & 258.2 \\
\hline 6 & 3 & 233.0456 & 1 & 195.5 & 3 & 235.9531 & 3 & 236.0 \\
\hline 7 & 1 & 253.2750 & 1 & 260.1 & 1 & 268.8635 & 1 & 269.0 \\
\hline 8 & 3 & 233.0456 & 3 & 234.3 & 3 & 235.9531 & 3 & 236.0 \\
\hline 9 & 1 & 320.3832 & 1 & 325.3 & 1 & 331.4877 & 1 & 331.6 \\
\hline \multirow[t]{2}{*}{10} & 1 & 239.3969 & 1 & 246.3 & 1 & 255.0562 & 1 & 255.2 \\
\hline & \multicolumn{4}{|c|}{ LOAD DEMAND = $2600 \mathrm{MW}$} & \multicolumn{4}{|c|}{ LOAD DEMAND = $2700 \mathrm{MW}$} \\
\hline \multirow[t]{2}{*}{ UNIT } & \multicolumn{2}{|r|}{ PM } & \multicolumn{2}{|c|}{ HM [9] } & \multicolumn{2}{|r|}{$\mathrm{PM}$} & \multicolumn{2}{|c|}{ HM [9] } \\
\hline & FT & GEN (MW) & FT & GEN (MW) & FT & GEN (MW) & FT & GEN (MW) \\
\hline 1 & 2 & 216.5442 & 2 & 216.4 & 2 & 218.2499 & 2 & 218.4 \\
\hline 2 & 1 & 210.6058 & 1 & 210.9 & 1 & 211.6626 & 1 & 211.8 \\
\hline 3 & 1 & 278.1441 & 1 & 278.5 & 1 & 280.7228 & 1 & 281.0 \\
\hline 4 & 3 & 239.0967 & 3 & 239.1 & 3 & 239.6315 & 3 & 239.7 \\
\hline 5 & 1 & 275.5154 & 1 & 275.4 & 1 & 278.4973 & 1 & 279.0 \\
\hline 6 & 3 & 239.0967 & 3 & 239.1 & 3 & 239.6315 & 3 & 239.7 \\
\hline 7 & 1 & 285.7585 & 1 & 285.6 & 1 & 288.5845 & 1 & 289.0 \\
\hline 8 & 3 & 239.0967 & 3 & 239.1 & 3 & 239.6315 & 3 & 239.7 \\
\hline 9 & 1 & 343.8134 & 1 & 343.3 & 3 & 428.5216 & 3 & 429.2 \\
\hline 10 & 1 & 271.5861 & 1 & 271.9 & 1 & 274.9667 & 1 & 275.2 \\
\hline
\end{tabular}

Table 2. Comparison of the total cost with different techniques.

\begin{tabular}{ccccc}
\hline \multirow{2}{*}{ TECHNIQUES } & \multicolumn{4}{c}{ TOTAL FUEL COST $(\$ / \mathrm{h})$} \\
\cline { 2 - 5 } & $2400 \mathrm{MW}$ & $2500 \mathrm{MW}$ & $2600 \mathrm{MW}$ & $2700 \mathrm{MW}$ \\
\hline HM [9] & 488.500 & 526.700 & 574.030 & 625.180 \\
HNN [10] & 487.870 & 526.130 & 574.260 & 626.120 \\
AHNN [11] & 481.700 & 526.230 & 574.370 & 626.240 \\
HGA [12] & 481.7226 & 526.2388 & 574.3808 & 623.8092 \\
MPSO [15] & 481.723 & 526.239 & 574.381 & 623.809 \\
TM [16] & 481.6 & ----- & ---- & 623.7 \\
IFEP [17] & ----- & 526.25 & ----- & ----- \\
FEP [17] & ----- & 526.26 & ----- & ---- \\
CEP [17] & ----- & 526.25 & ----- & ---- \\
PSO [18] & ----- & ---- & ---- & 623.88 \\
IEP [19] & 481.779 & 526.304 & 574.473 & 623.851 \\
PROPOSED & 481.7226 & 526.2338 & 574.0105 & 623.8092 \\
METHOD & &
\end{tabular}




\section{Conclusions}

The economic dispatch problem with multiple fuel options is a complex optimization problem whose importance may increase as competition in power generation intensifies. This paper presents economic dispatch problem with multiple fuel options using composite cost function. The proposed CCF based solution for economic dispatch with MFO offers a best contribution in the area of economic dispatch. In contrast to the HM [9], this approach fully explores the cost coefficients and gives a promising value of power for providing improved economic dispatch. The HM method requires the valid assumptions such as initial value of lambda and it iteratively solves the problem. The proposed methodology is a non-iterative method that directly gives the optimal generation schedule of the generating set and it does not require any assumptions. The numerical results demonstrate that the proposed approach offers a better convergence rate, minimum cost to be achieved and better solution than the existing methods. As power systems are usually large scale systems, the proposed method may be suggested for the solution of economic load dispatch problems and it is also suitable for online applications.

\section{Acknowledgements}

The authors gratefully acknowledge the authorities of Annamalai University, Annamalainagar, Tamilnadu, India, for their continued support, encouragement, and the extensive facilities provided to carry out this research work.

\section{References}

[1] A. J. Wood and B. F. Wollenberg, "Power Generation Operation and Control,” 2nd Edition, Wiley, New York, 1996.

[2] A. Chakrabarti and S. Halder, "Power System Analysis Operation and Control,” 3rd Edition, PHI, New Delhi, 2010.

[3] K. P. Wong and Y. W. Wong, "Genetic and Genetic/ Simulated-Annealing Approaches to Economic Dispatch," IEE Proceedings of Generation, Transmission and Distribution, Vol. 141, No. 5, 1994, pp. 507-513. doi: 10.1049/ip-gtd:19941354

[4] Z. L. Gaing, "Particle Swarm Optimization to Solving the Economic Dispatch Considering the Generator Constraints," IEEE Transactions on Power Systems, Vol. 18, No.3, 2003, pp. 1187-1195. doi: 10.1109/TPWRS.2003.814889

[5] B. K. Panigrahi, S. R. Yadav, S. Agrawal and M. K. Tiwari, "A Clonal Algorithm to Solve Economic Load Dispatch,” Electric Power Systems Research, Vol. 77, No.
10, 2007, pp. 1381-1389.

doi:10.1016/j.epsr.2006.10.007

[6] Cheng-Chien Kuo, "A Novel Coding Scheme for Practical Economic Dispatch by Modified Particle Swarm Approach," IEEE Transactions on Power Systems, Vol. 23, No. 4, 2008, pp. 1825-1835. doi: 10.1109/TPWRS.2008.2002297

[7] N. Noman and H. Iba, "Differential Evolution for Economic Dispatch Problems,” Electric Power Systems Research, Vol. 78, No. 8, 2008, pp. 1322-1331. doi:10.1016/j.epsr.2007.11.007

[8] B. K. Panigrahi and V. R. Pandi, "Bacterial Foraging Optimization: Nelder-Mead Hybrid Algorithm for Economic Load Dispatch,” IET Generation Transmission Distribution, Vol. 2, No. 4, 2008, pp. 556-565. doi:10.1049/iet-gtd:20070422

[9] C. E. Lin and G. L. Viviani, "Hierarchical Economic Dispatch for Piecewise Quadratic Cost Functions," IEEE Transaction on Power Application Systems, Vol. PAS-103, No. 6, 1984, pp. 1170-1175. doi:10.1109/TPAS.1984.318445

[10] J. H. Park, Y. S. Kim, I. K. Eom and K. Y. Lee, "Economic Load Dispatch for Piecewise Quadratic Cost Function using Hopfiled Neural Network,” IEEE Transactions on Power Systems, Vol. 8, No. 3, 1993, pp. 1030-1038. doi:10.1109/59.260897

[11] K. Y. Lee, A. Sode-Yome and J. H. Park, "Adaptive Hopfiled Neural Network for Economic Load Dispatch,” IEEE Transactions on Power Systems, Vol. 13, No. 13, 1998, pp. 519-526. doi:10.1109/59.667377

[12] S. Baskar, P. Subbaraj and M. V. C. Rao, "Hybrid Real Coded Genetic Algorithm Solution to Economic Dispatch Problem," Computers Electrical Engineering, Vol. 29, No. 3, 2003, pp. 407-419. doi:10.1016/S0045-7906(01)00039-8

[13] S. C. Lee and Y. H. Kim, “An Enhanced Lagrangian Neural Network for the ELD Problems with Piecewise Quadratic Cost Functions and Nonlinear Constraints," Electric Power Systems Research, Vol. 60, No. 3, 2002, pp. 167-177. doi:10.1016/S0378-7796(01)00181-X

[14] J.-R. Won and Y.-M. Park, "Economic Dispatch Solutions with Piecewise Quadratic Cost Functions Using Improved Genetic Algorithm,” Electric Power Energy Systems, Vol. 25, No. 5, 2003, pp. 355-361. doi:10.1016/S0142-0615(02)00098-4

[15] J.-B. Park, K.-S. Lee, J.-R. Shin and K. Y. Lee, “A Particle Swarm Optimization for Economic Dispatch with Nonsmooth Cost Functions," IEEE Transactions on Power Systems, Vol. 20, No. 1, 2005, pp. 34-42. doi: 10.1109/TPWRS.2004.831275

[16] D. R. Liu and Y. Cai, "Taguchi Method for Solving the Economic Dispatch Problem with Nonsmooth Cost Functions," IEEE Transactions on Power Systems, Vol. 20, No. 4, 2005, pp. 2006-2014. doi:10.1109/TPWRS.2005.857939

[17] T. Jayabarathi, K. Jayaprakash, D. N. Jayakumar and T. Raghunathan, "Evolutionary Programming Techniques for Different Kinds of Economic Dispatch Problems," 
Electric Power Systems Research, Vol. 73, No. 2, 2005, pp. 169-176. doi:10.1016/j.epsr.2004.08.001

[18] D. N. Jeyakumar, T. Jayabharathi and T. Raghunathan, "Particle Swarm optimization for Various Types of Economic Dispatch Problems," Electric Power Energy Systems, Vol. 28, No. 1, 2006, pp. 36-42. doi:10.1016/j.ijepes.2005.09.004

[19] Y.-M. Park, J.-R. Won and J.-B. Park, “A New Approach to Economic Load Dispatch Based on Improved Evolutionary Programming," Engineering Intelligent Systems Electrical Engineering Communication, Vol. 6, 1998, pp. 103-110. 